\title{
New Experience and Thought of Meticulous Painting and Teaching
}

\author{
Yuan Liu \\ Art College of Xi'an University of Arts and Science, 710065
}

\author{
Keywords: Meticulous painting; Color; Emotion
}

\begin{abstract}
Nowadays, due to the lack of Chinese painting test for the admission to art college, lots of students generally have a poor basis and understanding towards Chinese painting. Students do not have a comprehensive and deep understanding about meticulous painting which is an important branch of Chinese painting. This essay aims at analyzing some new experience and thought of meticulous painting from the point of teaching, and investigating some points on which need to be focus during the teaching of meticulous painting.
\end{abstract}

\section{New Experience of Meticulous Painting Teaching}

Development History of Meticulous Painting. Meticulous painting is the blend of ancient and modern art form, which becomes more and more popular and welcome. It begins to step into mainstream from margin in Chinese painting fields. The emergence of diversified art patterns, and the mutualistic form, style and genre all together urge the artist of meticulous painting to create the work with profound awareness, diversified concept and method, which is also the inevitable trend and characteristic when meticulous painting gets into modernity. The mutualism and diversity facilitate the prosperity of meticulous painting and create the new outlook of modern meticulous painting. Urban painting and ecologic painting emerge. Rural painting is infused with new factors. The updated thinking mode brings new concept to traditional landscape painting, bird-and-flower painting and figure painting. A number of painters' urge to create their works is creative idea, which becomes major trend of modern creation thought. Meticulous painting in China has been developed in a good pace. Painters hold their own unique style in terms of form language, and they pay too much attention to their actual life around. This creation attitude is the keystone for the prosperity of meticulous painting. In another way, painters focus on skill and artistry, and they also seek for creative inspiration from real life and their own inner world, which is not against the basic rules of art creation.

It is sure that the modern meticulous painting is confronted with some problems. For example, many painters focus more on skill and details, and there is improper application of texture processing. Painters get easily to ignore the freehand brushwork which has been emphasized by traditional art and culture thousand years ago, and this is exactly the desire for emotion and cultural deposits from the art work. Though it is a trend though of the above phenomenon, it will be a huge setback to the spirit of meticulous painting even of Chinese painting if it is greatly developed in the future. Therefore, During the teaching of meticulous painting, teachers should emphasize the spirit and emotion from the art work, try not to focus all the time on skills and ignore to seek for spirit and cultural deposits. Teachers should be the one who enable students' works to carry forward the tradition and yet blend with modern style. Except for imparting traditional creation method, teachers need to convey traditional art background and spirit content. And students will be influenced by what they constantly see and hear. All this aims to create the classroom atmosphere filled with art under which the students perceive the essence of traditional meticulous painting. Before they grow their own creation style, they should be taught to step out of the stereotyped art style. The silent and profound painting style from ancient painters could be a best reference for students. These ancient artists though consider that the elaborate skill is important, yet they still would not lose the interest. Therefore, students can take it as reference that the interest spirit of traditional art work, which creates the shape by imagination and brings organic vigor to the work. 
The traditional creation method enables the meticulous painting to be an art expression method with Chinese culture.

The Selection of Copy Reference. Before the teaching of meticulous painting, teachers devote most of the time on studying the basic copy skill, since copying is the basis to learn and grasp the meticulous painting. The emphasis of copying is on how to choose a regular and popular reference. In terms of teaching, the content of reference need to be accurate and flawless, by which the reference greatly plays its role and the students absorb the information in the greatest extent. What 's more, the information absorbed by students from reference is comprehensive, and it greatly enhances the aesthetic sense for the future studying. Therefore, during the teaching process, there are two factors need to be take into consideration, the copy reference could be classical and traditional, or either with modern style.

Meticulous painting plays an important role in Chinese painting. Especially the meticulous painting in five dynasties in Chinese history such Tang and song, is regulated with strict pattern. For example, artist at that time is very fastidious about the lines and color, their works are full of national features. The most effective way to learn is to copy, which is the truth for all of the students no matter how good or bad their basic skill is. The copying reference mentioned above could improve students' skill of meticulous painting. Students acquire the traditional culture from the reference. Constantly copying the proper reference could enhance and solidify students' basic skill. During the copying class, teachers could create more lectures about how to appreciate the work. Though the task of meticulous painting is to transmit the tradition, the copying and study for modern reference should not be ignored as well. During the teaching of art, the conflict and selection between tradition and modernism has always been controversial, and meticulous painting is involved too. Students live and study in modern society today, the aesthetic sense and cultural deposits are basically full with modern style. Therefore, both the development of modernism and the inheritance of traditional culture are of great significance, which makes it important to choose between tradition and modernism when selecting for copying reference. Teachers need to find a balance between them, so that acquaints the students to create the work with traditional beauty and modern style.

The Practice. In the industry of Chinese art education, the innovation and reformation of meticulous painting need to start with practice out of classroom. In the traditional class of meticulous painting, teachers focus mainly on the theory class and teaching within the classroom. A plenty of specialized lectures are accomplished in the classroom. The pass teaching concept believes in teaching in the room, and since it is the only way to teach, which ignores the significance to sketch the scenery out of classroom. Art lectures is different from other specialized lectures. The effect of practice is bigger than that of theory. In the limited teaching time, students can hardly improve their meticulous painting skills in the greatest extent. There are less specialized lectures in most of the university or college, more with the poor painting skills by students, teaching only in classroom can hardly improve their skills. Therefore, the setting of outdoor practice is necessary. During the creation in nature, it is easy to relax the students, and get them into the nature, which will be helpful for them to deeply understand how to express the content by a piece of work.

In another way, outdoor lectures can improve the practice ability of students. The teaching of art contains both the basic theory and knowledge, and the comprehensive ability. Most of the lecture setting in university or college are diffuse. Single method like teaching only theory or indoor can hardly acquaint the students with skills. There are still a number of problems even though lots of universities set up the outdoor practice, such as the frequency and duration of a class. These problems reduce the effect of outdoor practice. Students create in a limited short time and practice in a lower frequency. Creation needs either time or attention. Outdoor practice in a short time and lower frequency will only affect students in a bad way, since it reduces the confidence and passion for students to create the work. Therefore, how to set up proper outdoor practice is of great significance. For example, the location, frequency and the duration of one class, also the content and theme of the class could all be taken into consideration, which will one hundred percent enjoy the practice and benefits a lot, and by which the students can strongly improve their painting skills. 
The Sense of Spirituality. Color is normally considered as the essence to express the spirit. A work without spirit has no ornamental value, because it looks dull and bland. Spirit can make a work vivid. Painters with great spirit can make the work enjoyable, such as Xu Wenchang, his work is full of mountain stuff. Painters with bland spirit could create freehand work, like Chen Zhifo. However, the skills and wonderful thing about meticulous painting is how to handle your tools. The way you use the pen is the way you express your spirit. The way a painter use a pen represents his upbringings, personality, interest and skills. To better perform the skills and spirit by using a pen, the shape could be freehand or even changes. Therefore, proper using the pen could better express the spirit.

It is sure that the color is important even for using the ink to create. However, how to use the ink as color is a way to see how the painter handles the pen. If painters cannot even grasp how to paint the lines, then they should not be able to create meticulous painting. Because in relative terms, the lines of a meticulous painting is not as complicated as the lines of freehand work, but the skill is always required. The basis of meticulous painting is on the lines and shape, and the color is the most important factor. The color is also a way to see a painter's personality, spirit and skills. The color of meticulous painting cannot always follow the real color, if so, it is just a copy of the substance, which is the biggest problem of meticulous painting in the current situation. The meticulous painting in Song dynasty tends to be thick and profound. Therefore, though the meticulous painting is based on the freehand work, but it is not about real. To be thick, profound, bland, or enjoyable is something that the meticulous painters always seek for, which is so-called spirit. In Chen Zhifo's work, he absorb some characteristics of Japanese work, and also with pattern. Relatively speaking, his work is full of creation, and with high level and high accomplishment.

\section{Thought of Meticulous Painting Teaching}

The Color of Meticulous Painting. If we envision that the line of meticulous painting is the keystone of a house, then the color of meticulous painting is the decoration of this house. The final outlook of this house is all about the decoration. The selection of color in meticulous painting is different with ink and wash painting, and it is significant. The ink used for ink and wash painting is not just black. The relationship of ink and color flickers a sort of cultural characteristics and philosophical thought. In 21 century, a number of challenge and innovation blocked in front of the development of Chinese painting. Many works failed to satisfy the constantly increasing aesthetical standard and need by modern people. Therefore, the innovation and reformation of those Chinese painting are all about the breakthrough of the aesthetical sense and selection of color. And how to add with different element and color into the teaching is one of the biggest challenge confronting the modern teachers.

In terms of that challenge and the need of innovation and reformation, Chinese painting cannot only seek for modernism. During the daily teaching, teachers keep digging and delivering the resource of traditional meticulous painting, analyze the essence of color used in these traditional works. In practice class, traditional works are applied as the copying reference, which enables the students to absorb the color application in traditional history whose characteristics of color greatly express the spirit. In another way, after students have a deep understanding of traditional color and solidify the basic skills, teachers can properly inspire and encourage the students to blend the traditional color into the modern creation. Under the conflict of tradition and modernism, the transition of meticulous painting from tradition and modernism can be accomplished.

Except for mutual transition and blend of tradition and modernism, we can take western paintings as a reference. For example, we can analyze the color and appreciate from the western works and the painting tools and pigment used in western works can be learned as well, such as cosmetic pigment and watercolor. It is necessary to encourage the students to blend the color that they acquire into the creation of meticulous painting. It is worth mentioning that, during the transmit and blend process of tradition to modernism, and also taking western works as reference, the solid meticulous painting skill is the most crucial premise. Otherwise, after reference and transmit, the final work can hardly represent the essence of Chinese painting, which means that the 
traditional culture, spirit and style are missing. Therefore, during the teaching process, teachers should focus on the practice of basic skills like copying at the first place, and then encourage the students to blend the tradition and modernism, and take the western color application as example.

Devotion of Emotion. Cihai has explained that the so-called emotion means the psychological performance of people's joy, anger, sadness and happiness, and that emotion has been developed from learning and changing the world. The emotion performance has always been the gist of art. Art activity denotes emotion activity. In addition, art creation is the product of emotion. Therefore, art is a single and dependent subject, which especially focuses on the investigation of people's emotion. All the changing in inner world such as joy, anger, sadness and happiness are the entire object for artist to study and pay attention to.

Teaching mode of meticulous painting is to acquaint the students with basic skills. However, abstract things such as emotion and art come from people's sensation and life, which cannot be acquired by only taking the class in the room. A successful work can show people the emotion that he or she put into, in another way, people see how these artists understand and perform the art. Therefore, during the teaching, expect for the appreciation and analysis, copying of the work, students should be taught to appreciate the painter, and try to understand the latent emotion and thought the painters try to tell. Through the analysis of painters' emotion, students should acquire how to turn every single element from the work into emotion, give the work a life and spirit. Different timing, emotion and scene, also the experience could bring different emotion to the work. Teachers should aperiodically demonstrate different works to students. Some works need to be feel more than once before understanding the emotion. And some other works give different feeling to students no matter how many times they see the work. Students should be encouraged to boldly guess what the works try to tell, guess what the emotion the painters express. Keep practicing like this can improve the sensibility and creation thought. Such as Lin Daiyu in classical novel A Dream in Red Mansions, she is a typical figure who treats everything with strong mind and emotion. We are not here to encourage to create the work under sad emotion, what we try to encourage is to train the students a habit to feel and express. Painter, as an art worker, his sensibility and emotion need to be strong. Solid basic skills can make a student a qualified painter. However, viewing things with strong emotion and great sensibility on art can make a student an excellent painter. During the teaching process, teachers can train the students to record the feeling and emotion that they have towards any substance, such as the scenery, mountain, trees and so on, which could be the inspiration of creation in the future. The accumulation of idea and inspiration is the gift for an artist.

The Transmit of Tradition. Meticulous painting is a branch of Chinese painting, which is a kind of inheritance to the quintessence of Chinese culture and Chinese traditional culture as well. During the creation of traditional painting, teachers should help the students to find out and transmit more meticulous painting skills, so traditional works can be taken as an example. Before deep understanding and investigation of traditional works, teachers absorb useful information, for example, when creating ink and wash painting, teachers apply the theory of calligraphy, and show how to perform the work more soft and beautiful with the shape of the nib. Ink can also emit vivid art sense by the work. Through the shape theory and the application of the nib, the ink and wash painting blend the abstract object with half-abstract object, which enhances the freehand features, objective, emotional and vivid sense to the work. The fiction and fact transmit and the variety level can be diversified by using the ink, water and the nib skills. All this aims to seek for the freehand feature, focuses on emotion and seeks for artistic conception. The art language can be broader and diversified by the emotion and spirit of your work. In addition, the meticulous painting focuses on the object significance as well. Teachers can take the fresco, printmaking, Chinese new year poster and industrial art as reference to diversify the content of meticulous painting. Therefore, the inner transmit, reference and absorption to traditional artwork enable the meticulous painting to inherit the Chinese poem, which expresses a kind of emotion and artistic conception. Meticulous painting with its inheritance towards tradition constantly transcends the single language mode of the classic meticulous painting such as contouring and rendering in multiple times, it finds its way to broaden its technical language from the legacy, yet still looks for modern painting significance and the 
developing chances. It creates the modern significance based on the history and makes it successful to transmit the traditional painting to modern meticulous painting.

\section{Conclusion}

In today's teaching situation of meticulous painting, the main problem confronting the teachers is how to let the student have a deep understanding towards meticulous painting, which includes the understanding of developing history, the practice of basic skills, the practice ability outdoor, the inspiration of spirit, the significance of color, how to express the emotion by the work, how to absorb the feeling and emotion towards anything substance in daily life, the art inspiration, and how to create the work with the accumulated inspiration and idea. And it is of great importance that the most significant point for teaching meticulous painting is how to let the modern students to better inherit the tradition.

\section{Reference}

[1] Q.Y.Zheng: Theory study of Chinese traditional art education, Vol. 4 (2004) No.4

[2] Y.M.Hu: Emotional function in teaching art in school, Vol. 5 (2005) No.5

[3] H.Y.Wei: Creation and teaching of college about Chinese painting, Vol. 5 (2007) No.5

[4] Z.H.Li: Beauty history, Vol. 5 (2001) No.5

[5] J.X.Peng: General overview of art, Vol. 12 (2002) No.1

[6] H.X.Wang: Though and strategy of art education in university, Vol. 8 (2005) No.7

[7] Q.Gao: The lack of Chinese traditional culture in Chinese painting teaching, Vol. 11 (2012) No.5, p.86-8

[8] Y.C.Pan: Modern art education history in China, Vol. 7 (2002) No.2 